\title{
Diet cost, diet quality and socio-economic position: how are they related and what contributes to differences in diet costs?
}

\author{
Petra J Rydén* and Linda Hagfors \\ Department of Food and Nutrition, Umeå University, S-90187 Umeå, Sweden
}

Submitted 26 0ctober 2010: Accepted 23 November 2010: First published online 24 January 2011

\begin{abstract}
Objective: To examine diet costs in relation to dietary quality and socio-economic position, and to investigate underlying reasons for differences in diet costs.

Design: Dietary intake was assessed by a $4 \mathrm{~d}$ food diary and evaluated using the 2005 Healthy Eating Index (HEI). National consumer food prices collected by Statistics Sweden and from two online stores/supermarkets were used to estimate diet costs.

Setting: Sweden.

Subjects: A nationally representative sample of 2160 children aged 4, 8 or 11 years. Results: Higher scores on the HEI resulted in higher diet costs and, conversely, higher diet costs were linked to increased total HEI scores. Children who consumed the most healthy and/or expensive diets ate a more energy-dilute and varied diet compared with those who ate the least healthy and/or least expensive diets. They also consumed more fish, ready meals and fruit. Regression analysis also linked increased food costs to these food groups. There was a positive, but weak, relationship between HEI score and diet cost, parental education and parental occupation respectively.

Conclusions: Healthy eating is associated with higher diet cost in Swedish children, in part because of price differences between healthy and less-healthy foods. The cheapest and most unhealthy diets were found among those children whose parents were the least educated and had manual, low-skill occupations. Our results pose several challenges for public health policy makers, as well as for nutrition professionals, when forming dietary strategies and providing advice for macro- and microlevels in society.
\end{abstract}

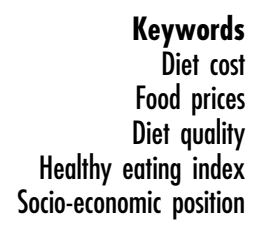

Diet cost

Food prices

Healthy eating index

Socio-economic position
The disparities in health between groups with high and low socio-economic position (SEP) ${ }^{(1)}$ are also apparent in their diets. High-SEP groups have healthier intakes of key nutrients ${ }^{(2,3)}$, higher intakes of fruit and vegetables ${ }^{(2,4,5)}$ and healthier dietary patterns ${ }^{(2,6-8)}$ than low-SEP groups. One of the reasons for the inequity in dietary intake might be the cost of healthy eating ${ }^{(9)}$.

There is increasing knowledge about the relationship between consumer food costs and diet. In theory, it is quite possible to compose a nutritious diet at low $\operatorname{cost}^{(10,11)}$. However, these theoretical diets do not always correspond to real-life situations ${ }^{(12,13)}$. Generalized models of healthy diets, planned on a food group level, have been shown to be too expensive for low-SEP families ${ }^{(14,15)}$. When comparing costs of consumed diets, studies show somewhat mixed results: cross-sectional dietary surveys often show that healthier diets cost more ${ }^{(16-24)}$ while in intervention studies, on the other hand, the healthy diets are often less expensive than the control diet ${ }^{(25-28)}$; although contradicting examples can be found ${ }^{(29,30)}$.
Although accumulating evidence indicates that healthy eating is more expensive, the need for additional studies of consumed diets is apparent. An intriguing question that has not yet been fully answered is why a healthier diet costs more. Energy-dense foods, which contribute to a less-healthy diet if eaten in excess ${ }^{(31)}$, are often cheaper ${ }^{(32)}$; however, the complexity of dietary behaviour implies that this is not the only reason for differences in diet costs. It is also important to investigate costs in different parts of the world, because food prices and dietary habits differ among countries $^{(33)}$. Few studies have examined the relationships among diet, cost and socio-economic variables.

The aim of the present study was to examine diet costs in relation to dietary quality and SEP in Swedish children. We also investigated reasons for differences in diet costs by relating cost to intake on a food group level. To our knowledge, no other studies in this area have been conducted using the dietary intake of children. If healthy food cost is related to SEP, it is an important incentive for updated public health policies. 


\section{Materials and methods}

Dietary intake data were collected in 2003 in a Swedish national food survey called 'Riksmaten - children'(34) The population consisted of randomly selected children, who were 4,8 or 11 years old, from a stratified sample of municipalities representative of Sweden. The stratification considered regional differences and parents' income and education. The 4-year-old children were selected individually, while the 8- and 11-year-olds were included classwise (grades 2 and 5) from randomly selected schools. In total, 3423 children were sampled (924 children aged 4 years, 1209 children from grade 2, 1290 children from grade 5). Of these children, 3055 (89\%; 823, 1070 and 1162 children who were 4,8 and 11 years old, respectively) agreed to participate, and 2535 (74\%; 590, 909 and 1036 children who were 4, 8 and 11 years old, respectively) completed the study. Forty-one children were excluded due to incomplete dietary data, leaving a final population of 2494 children ( $49 \%$ girls).

\section{Assessment of dietary intake, BMI and sociodemographic factors}

Dietary assessments were made using open, estimated food diaries covering four consecutive days. All days of the week were evenly represented. Parents or other caregivers were responsible for the diaries of the 4-yearolds, whereas the schoolchildren registered their dietary intake by themselves or with assistance from an adult (parent and/or teacher). Specially trained informers visited the families of each 4-year-old to inform them about the survey and how to complete the food diary. Information was provided to the schoolchildren by informers who visited teachers and children in the selected classes. The food diaries were designed to be suitable for the different age groups and contained written instructions regarding the dietary assessment. During the four recording days, all foods, beverages and supplements were registered. Consumed amounts were estimated in household measures or by comparisons with a book containing pictures of different portion sizes.

The estimated intakes of energy and nutrients (including supplements) were calculated using the nutritional analysis package MATs version 4.03 (Rudans Lättdata, Västerås, Sweden) based on the Swedish National Food Administration's food composition database (version $04 \cdot 1 \cdot 1$ ). In addition to the food diary, a questionnaire containing questions about the child's weight and height and parents' education and occupation was answered. BMI was calculated from self-reported weight and height. The validity of reported energy intake was evaluated by comparing the ratio between reported energy intake (EI) and BMR with the lowest ratio plausible (EI:BMR $=1 \cdot 06$ ) for a dietary assessment over $4 \mathrm{~d}^{(35)}$. The BMR was estimated according to standard equations using body weight, age and sex ${ }^{(36)}$. If data regarding weight were missing, the BMR was calculated using ageand sex-adjusted group means. Of 2494 children, 334 $(13 \cdot 4 \%)$ were classified as under-reporters and hence excluded from the analysis, leaving a total of 2160 children.

\section{Measures of dietary quality}

The 2005 Healthy Eating Index (HEI) was used to assess the healthiness of the dietary intakes ${ }^{(37)}$. In short, HEI accounts for both food and nutrient intakes and includes adequacy components as well as moderation components (Table 1). Using twelve components, a total HEI score ranging from 0 to 100 is calculated, with a higher score indicating a healthier diet. An advantage of HEI is the energy adjustment (all components are calculated per $4184 \mathrm{~kJ}(1000 \mathrm{kcal}))$, which makes it possible to evaluate diet quality while controlling for diet quantity, important when studying children of different ages.

Minor adjustments were made for the solid fat, (alcohol) and added sugar component; fat levels above the Swedish 'Keyhole Symbol' (indicating healthier alternatives within a food group ${ }^{(38)}$ ) were considered as excess fat for the milk and meat and beans components, instead of the original threshold 'lowest fat form,'(39). In addition, added sugar was calculated using sucrose content, as specified in the Swedish Food Database ${ }^{(40)}$.

Total HEI score can be used to classify diets as 'poor' (total HEI score <50), 'needs improvement' (score 50-80) or 'good' (score $>80)^{(41)}$. However, because only three children had a total HEI score above $80(0 \cdot 1 \%)$, diets with an HEI score $>70$ were classified as high-HEI diets.

Energy density $(\mathrm{MJ} / \mathrm{g})$ was calculated including beverages. Food intake variation was defined as the number of unique food items reported during the data collection period, disregarding the amount consumed.

\section{Food price information}

Food price data were compiled from national average prices collected by Statistics Sweden for 391 out of a total of 991 food items covering $71 \%$ of the food intake. Prices for the remaining 600 food items were collected mainly from one online supermarket and one online grocery store. Both had the same prices online as in their physical store, but average prices were significantly higher at the grocery store. Hence, because the supermarket was part of a brand chain holding approximately $50 \%$ of the market share ${ }^{(42)}$ and a majority of Swedish families with children shop at supermarkets ${ }^{(43)}$, average prices were weighted 70/30 towards supermarket prices. Prices for meals from restaurants and fast foods were collected from Statistics Sweden and fast-food restaurants. Total food costs are presented as $€ / 4184 \mathrm{~kJ}(1000 \mathrm{kcal})$.

All of the prices were collected in the spring of 2010. This could impose seasonal differences in the prices of fruits and vegetables. However, the average prices of fruit and vegetable staples have low seasonal variability in Sweden, with the exception of harvest season in late 
summer/early autumn. No dietary data were collected during this period.

The amount of food consumed was recalculated into the amount of food purchased using factors adjusting for waste and water retention/loss during cooking (e.g. $100 \mathrm{~g}$ of banana eaten was multiplied by a factor of 1.59 (representing the weight of the banana peel), resulting in $159 \mathrm{~g}$ of banana purchased).

Each food item was classified into a food group (Table 2). Subgroups were created for food groups in which healthier options were available. These reflect the subcategories in HEI (i.e. healthier option in the fruit group is the same as the whole-fruit category in HEI).
In HEI, some categories are not mutually exclusive; i.e. the fatty part of a sausage is counted in the solid fat, alcohol and added sugar category, while the sausage as a whole is counted in the meat and beans category. For the food group classification, each food is represented in one food group only and, where applicable, in a subgroup. The food groups were used to assess costs as percentage of total costs, costs per $100 \mathrm{~g}$, and number of unique food items within groups.

\section{Statistical analysis}

All statistical analyses were performed using PASW Statistics statistical software package version $18 \cdot 0$ (SPSS Inc., Chicago,

Table 1 Healthy Eating Index-2005 components and scoring, adopted from Guenther et al. ${ }^{(37)}$

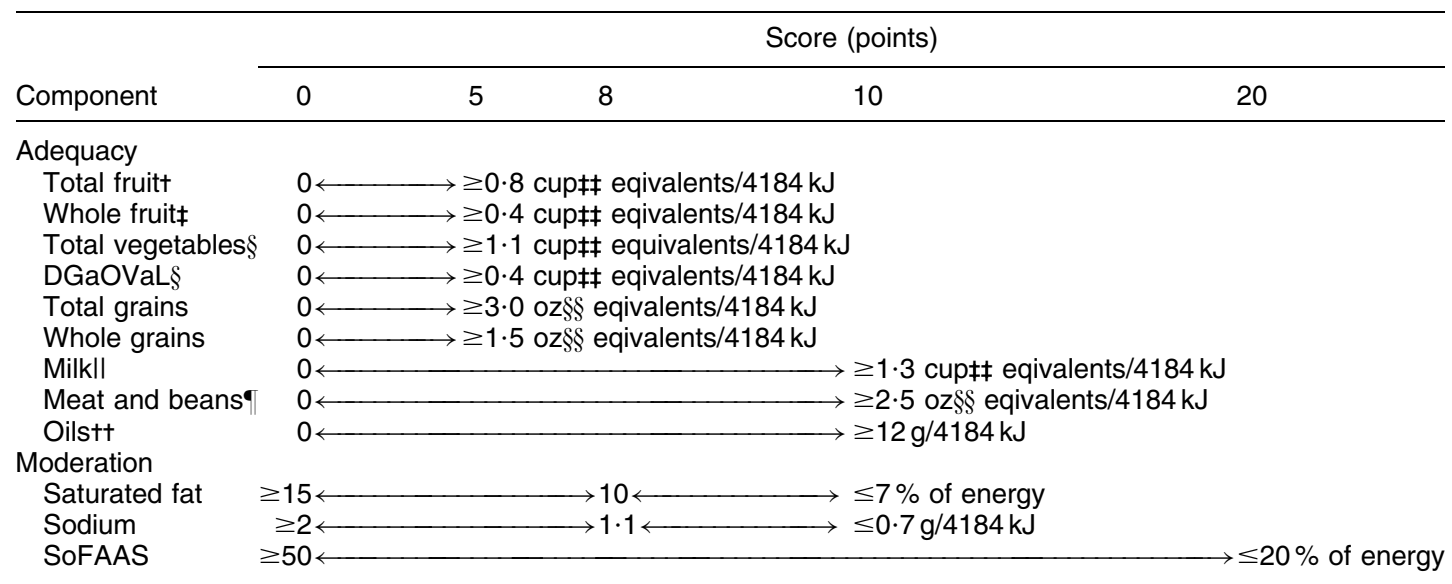

DGaOVaL, dark green and orange vegetables and legumes; SoFAAS, solid fats, alcoholic beverages and added sugars.

tIncludes $100 \%$ juice.

łlncludes all forms except juice.

§Includes legumes only after meat and beans standard is met.

IIIncludes all milk products such as fluid milk, yoghurt and cheese, and soya beverages.

- Includes legumes only if the meat and beans standard is otherwise not met.

ttIncludes non-hydrogenated vegetable oils and oils in fish, nuts and seeds.

$\$ ¥ 1$ cup = approx. $237 \mathrm{ml}$.

$\S \S 1 \mathrm{oz}=$ approx. $28.4 \mathrm{~g}$.

Table 2 Definition of food groups and subgroups used to analyse differences in cost

\begin{tabular}{|c|c|}
\hline Food group & Description \\
\hline $\begin{array}{l}\text { Fruit } \\
\text { Healthier option }\end{array}$ & $\begin{array}{l}\text { Fruit, berries, fruit juice } \\
\text { Fruit, berries }\end{array}$ \\
\hline Vegetables & Vegetables, potatoes, legumes, nuts, seeds, processed vegetable products \\
\hline Healthier option & Dark green and orange vegetables and legumest \\
\hline Grains & Bread, pasta, rice, porridge, breakfast cereals, grains \\
\hline Healthier option & Wholegrain products and grain products labelled with the Swedish 'Keyhole' indicating partial wholegrain content \\
\hline Dairy & Milk, milk products, cheese, eggs \\
\hline Healthier option & Milk, milk products and cheese labelled with the Swedish 'Keyhole' indicating low fat content, eggs \\
\hline Fish & Fish, shellfish, processed fish products \\
\hline Poultry & Poultry, processed poultry products (i.e. sausages, meatballs, cold cuts) \\
\hline Meat & Meat, game, offal, processed meat products (i.e. sausages, meatballs, cold cuts) \\
\hline Healthier option & Meat, game, offal, processed meat products labelled with the Swedish 'Keyhole' indicating low fat content \\
\hline Fat & Oil, butter, margarine, dressings, mayonnaise \\
\hline Healthier option & Oil, oil-based margarines, oil-based dressings, mayonnaise \\
\hline Miscellaneous & Salt, spices, baking ingredients (not flour), ketchup, mustard, beverages not included in other groups \\
\hline Ready meals & Ready-made meals, fast-food meals, meals at restaurants \\
\hline Discretionary calories & Sweets, snacks (incl. crisps, popcorn), ice cream, desserts, soft drinks, jam, marmalade, sugar \\
\hline
\end{tabular}

tAs specified in Healthy Eating Index-2005(37). 
IL, USA). When comparing groups, the Student $t$ test for independent samples, the Mann-Whitney $U$ test, one-way ANOVA, the Kruskal-Wallis test or the $\chi^{2}$ test was used. Post hoc tests were performed using the Tukey HSD test for ANOVA and the Dunn test for Kruskal-Wallis. Multivariate analyses were made using analysis of covariance for adjusted differences between socio-economic variables and standard linear multiple regression to assess relationships between total dietary cost and food groups. $P$ values below $0 \cdot 05$ (two-sided) were considered to be statistically significant.

Effect size was calculated to assess the magnitude of significant differences by using $\eta^{2}$ for the $t$ test and ANOVA, and $\phi$ or Cramer's $V$ for $\chi^{2}$. Low effect size is stated in the Results section.

\section{Results}

The average total HEI score for the whole group was $59 \cdot 99$ (SD 7•80). Children with a total HEI score below 50 ( $n$ 221, $10 \cdot 2 \%$ ) formed the low HEI-score group. Correspondingly, those with a total HEI score above 70 ( $n$ 202, 9.4\%) formed the high HEI-score group (Table 3). The high HEI-score group had a more favourable dietary intake when comparing individual nutrients, energy density and HEI component scores with those of the low HEI-score group.

Energy-adjusted costs showed that the diet of the high HEI-score group was more expensive (mean difference $€ 0 \cdot 34 / 4184 \mathrm{~kJ}$, SD $0 \cdot 018, P<0 \cdot 001$; Table 3 ). The largest proportion of the cost was placed on meat (17.3\%) in the high HEI-score group and on discretionary calories (25.4\%) in the low HEI-score group (Fig. 1). The high HEIscore group had higher average costs for fruits, vegetables, fish, poultry and grains, while the low HEI-score group had higher costs for discretionary calories, ready meals, miscellaneous and fat $(P<0 \cdot 001$, except for fat $P=0 \cdot 003)$. Dividing food groups into subgroups based on healthier options (as defined in Table 2) emphasized the differences in costs between the high- and low HEI-score groups, with the high HEI-score group having higher costs in all of the healthier food groups $(P<0 \cdot 001)$.

Dividing the participants into quintiles based on dietary costs revealed the same pattern: spending more money on food resulted in higher total HEI scores (mean difference in HEI score, lowest $v$. highest: $4 \cdot 85$, SD $0 \cdot 15, P<0 \cdot 001$; Table 4). Energy density decreased with rising food costs $(P<0 \cdot 001)$. Post boc tests revealed that those in the highest cost quintile consumed more fish, poultry, fruit, ready meals, vegetables, meat and miscellaneous products than those in the lowest cost quintile $(P<0 \cdot 001$ to $P=0 \cdot 007)$, while those in the lowest cost quintile consumed more dairy products $(P<0 \cdot 001)$, expressed as grams per $4184 \mathrm{~kJ}$. When comparing nutrient intakes, the highest cost quintile generally had a more favourable nutrient profile, with the exception of a lower intake of $\mathrm{Ca}$ and a higher intake of $\mathrm{Na}(P<0 \cdot 001$ to $P=0 \cdot 047)$; however, the magnitude of the differences was low, except for protein (percentage of energy), fibre and $\mathrm{Na}$ (data not shown).

\section{Constitutions of differences in food costs}

The highest cost quintile consumed a more varied diet (number of unique foods, Q5 $v$. Q1: $54 \cdot 20 v$. 45.98, $P<0 \cdot 001$; Table 4), as did the high HEI-score group (high HEI $v$. low HEI: $54 \cdot 02 v .47 \cdot 23, P<0 \cdot 001$; Table 3). In both comparisons, vegetables and fruit accounted for about half the difference in variety (data not shown).

A standard multiple regression was performed with total cost per $4184 \mathrm{~kJ}$ as the dependent variable and consumed amount of food $(\mathrm{g} / 4184 \mathrm{~kJ})$ from the different food groups as the independent variables. The adjusted $R^{2}$ value of 0.465 indicates that less than half of the variability in costs was explained by the amount of food ingested from various food groups (Table 5). However, fish, meat, fruit and ready meals together accounted for just over half of the variability in total cost (sum of unique variability $=0.586$ ), implying that these food groups are the major contributors to the differences between highand low-cost diets. The regression also implies that if, for example, fish intake increases by $10.92 \mathrm{~g}$ (1 SD), total food cost is likely to increase by $€ 0 \cdot 20$ ( $\beta$ for fish $(0 \cdot 453$ ) multiplied by SD for total cost $(€ 0 \cdot 45))$.

By comparing cost per $100 \mathrm{~g}$ from different food groups among different cost quintiles, we determined that the highest cost quintile consumed more expensive foods within almost all food groups (Table 4). The largest differences between the highest and lowest cost quintiles were found for fish (mean difference $€ 0.52$, SD $0 \cdot 04, P<0 \cdot 001$ ), ready meals (mean difference $€ 0 \cdot 50$, SD $0 \cdot 35, P<0 \cdot 001$ ) and meat (mean difference $€ 0 \cdot 16$, SD $0 \cdot 05, P<0 \cdot 001$ ).

\section{Socio-economic position in relation to 2005 Healtby Eating Index and cost}

There were significant differences in both total HEI score and total cost in relation to parental education and occupation, but the magnitude of the differences was low (Table 6). Children whose parents had a university degree ( $n$ 1023) had a total HEI score that was $4 \cdot 25$ points higher on average (SD $0 \cdot 61, P<0 \cdot 001$ ) and they consumed a more expensive diet (mean difference $€ 0 \cdot 17 / 4184 \mathrm{~kJ}$, SD $0 \cdot 02, P=0 \cdot 009)$ than children with less-educated parents (primary school, $n 73$ ). Differences among occupational levels were smaller but still significant for both total HEI score $(P=0 \cdot 001)$ and cost $(P=0 \cdot 001)$. The differences remained after adjusting for age and cost or total HEI score, but were weakened slightly.

\section{Discussion}

The present study showed that higher dietary costs were associated with healthier eating in Swedish children. 
Table 3 Descriptive characteristics of groups with low, intermediate and high total HEI score

\begin{tabular}{|c|c|c|c|c|c|c|c|c|}
\hline & & \multicolumn{2}{|c|}{$\mathrm{HEI}<50(n 221)$} & \multicolumn{2}{|c|}{$\mathrm{HEI}=50-70(n 1737)$} & \multicolumn{2}{|c|}{$\mathrm{HEI}>70(n 202)$} & \multirow[b]{2}{*}{$P$ value } \\
\hline & & Mean or Median & SD or P25-P75 & Mean or Median & SD or P25-P75 & Mean or Median & SD or P25-P75 & \\
\hline Total cost $(€ / 4184 \mathrm{~kJ})$ & $\begin{array}{l}\text { Mean, SD } \\
\text { Median, P25-P75 }\end{array}$ & $\begin{array}{l}2 \cdot 21 \\
2 \cdot 18\end{array}$ & $\begin{array}{c}0.39 \\
1 \cdot 95-2 \cdot 45\end{array}$ & $\begin{array}{l}2 \cdot 38 \\
2 \cdot 33\end{array}$ & $\begin{array}{c}0.45 \\
2.07-2.64\end{array}$ & $\begin{array}{l}2.55 \\
2.50\end{array}$ & $\begin{array}{l}0.41 \\
2.28-2.85\end{array}$ & $<0.001 \ddagger$ \\
\hline Boys (\%) & & $52 \cdot 5$ & & $50 \cdot 7$ & $2.01-2.04$ & $52 \cdot 0$ & & $0.849 \S$ \\
\hline Age (years) & $\begin{array}{l}\text { Mean, SD } \\
\text { Median, P25-P75 }\end{array}$ & $\begin{array}{c}9 \cdot 51 \\
11 \cdot 2\end{array}$ & $\begin{array}{c}2 \cdot 72 \\
8 \cdot 51-11 \cdot 7\end{array}$ & $\begin{array}{l}8 \cdot 54 \\
8 \cdot 87\end{array}$ & $\begin{array}{c}2 \cdot 89 \\
7 \cdot 98-11 \cdot 5\end{array}$ & $\begin{array}{l}7 \cdot 25 \\
8 \cdot 41\end{array}$ & $\begin{array}{c}2 \cdot 78 \\
4 \cdot 37-9 \cdot 16\end{array}$ & $<0.001 \ddagger$ \\
\hline ISO-BMI > 25 kg/m² (\%)† & & $12 \cdot 9$ & & $15 \cdot 8$ & & $21 \cdot 5$ & & $0.026 \S$ \\
\hline Food intake level (El:BMR) & $\begin{array}{l}\text { Mean, SD } \\
\text { Median, P25-P75 }\end{array}$ & $\begin{array}{l}1.63 \\
1.56\end{array}$ & $\begin{array}{c}0 \cdot 32 \\
1 \cdot 25-1 \cdot 81\end{array}$ & $\begin{array}{l}1.59 \\
1.49\end{array}$ & $\begin{array}{c}0 \cdot 32 \\
1 \cdot 22-1 \cdot 74\end{array}$ & $\begin{array}{l}1.57 \\
1.49\end{array}$ & $\begin{array}{l}0.29 \\
1 \cdot 3-1 \cdot 71\end{array}$ & $0.025 \ddagger$ \\
\hline Energy density (kJ/g) & $\begin{array}{l}\text { Mean, SD } \\
\text { Median, P25-P75 }\end{array}$ & $\begin{array}{l}4 \cdot 76 \\
4 \cdot 64\end{array}$ & $\begin{array}{c}0 \cdot 90 \\
4 \cdot 06-5 \cdot 17\end{array}$ & $\begin{array}{l}4 \cdot 25 \\
4 \cdot 19\end{array}$ & $\begin{array}{c}0.64 \\
3 \cdot 79-4 \cdot 64\end{array}$ & $\begin{array}{l}3 \cdot 81 \\
3 \cdot 79\end{array}$ & $\begin{array}{c}0.45 \\
3 \cdot 39-4 \cdot 05\end{array}$ & $<0.001 \ddagger$ \\
\hline Variation (no. of unique foods) & $\begin{array}{l}\text { Mean, SD } \\
\text { Median, P25-P75 }\end{array}$ & $\begin{array}{l}47 \cdot 2 \\
45 \cdot 0\end{array}$ & $\begin{array}{c}11 \cdot 0 \\
37 \cdot 0-54 \cdot 0\end{array}$ & $\begin{array}{l}51 \cdot 5 \\
50 \cdot 0\end{array}$ & $\begin{array}{c}10 \cdot 7 \\
42 \cdot 0-58 \cdot 0\end{array}$ & $\begin{array}{l}54 \cdot 0 \\
53 \cdot 0\end{array}$ & $\begin{array}{c}9 \cdot 84 \\
45 \cdot 0-60 \cdot 0\end{array}$ & $<0.001 \ddagger$ \\
\hline Protein (\%E) & $\begin{array}{l}\text { Mean, SD } \\
\text { Median, P25-P75 }\end{array}$ & $\begin{array}{l}13 \cdot 9 \\
13 \cdot 9\end{array}$ & $\begin{array}{c}2 \cdot 36 \\
12 \cdot 5-15 \cdot 7\end{array}$ & $\begin{array}{l}15 \cdot 1 \\
15 \cdot 2\end{array}$ & $\begin{array}{c}2 \cdot 31 \\
13 \cdot 6-16 \cdot 9\end{array}$ & $\begin{array}{l}15 \cdot 6 \\
15 \cdot 7\end{array}$ & $\begin{array}{c}2 \cdot 13 \\
14 \cdot 4-17 \cdot 1\end{array}$ & $<0.001 \ddagger$ \\
\hline Fat (\%E) & $\begin{array}{l}\text { Mean, SD } \\
\text { Median, P25-P75 }\end{array}$ & $\begin{array}{l}34 \cdot 4 \\
34 \cdot 0\end{array}$ & $\begin{array}{c}4 \cdot 44 \\
31 \cdot 2-37 \cdot 3\end{array}$ & $\begin{array}{l}31 \cdot 6 \\
31 \cdot 4\end{array}$ & $\begin{array}{c}4 \cdot 17 \\
28 \cdot 8-34 \cdot 1\end{array}$ & $\begin{array}{l}28 \cdot 5 \\
28 \cdot 6\end{array}$ & $\begin{array}{c}4 \cdot 12 \\
25 \cdot 6-31 \cdot 1\end{array}$ & $<0.001 \ddagger$ \\
\hline Carbohydrates (\%E) & $\begin{array}{l}\text { Mean, SD } \\
\text { Median, P25-P75 }\end{array}$ & $\begin{array}{l}51 \cdot 7 \\
51 \cdot 7\end{array}$ & $\begin{array}{c}5 \cdot 22 \\
47 \cdot 8-55 \cdot 5\end{array}$ & $\begin{array}{l}53 \cdot 2 \\
53 \cdot 1\end{array}$ & $\begin{array}{c}4 \cdot 98 \\
49 \cdot 8-56 \cdot 4\end{array}$ & $\begin{array}{l}55 \cdot 8 \\
55 \cdot 7\end{array}$ & $\begin{array}{c}4 \cdot 59 \\
52 \cdot 6-59 \cdot 0\end{array}$ & $<0.001 \ddagger$ \\
\hline Fibre $(g / 4184 \mathrm{~kJ})$ & $\begin{array}{l}\text { Mean, SD } \\
\text { Median, P25-P75 }\end{array}$ & $\begin{array}{l}5 \cdot 82 \\
5 \cdot 77\end{array}$ & $\begin{array}{c}1 \cdot 50 \\
4 \cdot 96-6 \cdot 60\end{array}$ & $\begin{array}{l}7 \cdot 27 \\
7 \cdot 21\end{array}$ & $\begin{array}{c}1 \cdot 55 \\
6 \cdot 18-8 \cdot 21\end{array}$ & $\begin{array}{l}9 \cdot 26 \\
8.95\end{array}$ & $\begin{array}{c}1 \cdot 90 \\
7 \cdot 89-10 \cdot 5\end{array}$ & $<0.001 \ddagger$ \\
\hline Sucrose (\%E) & $\begin{array}{l}\text { Mean, SD } \\
\text { Median, P25-P75 }\end{array}$ & $\begin{array}{l}15 \cdot 7 \\
15 \cdot 3\end{array}$ & $\begin{array}{c}4 \cdot 77 \\
12 \cdot 3-18 \cdot 5\end{array}$ & $\begin{array}{l}13 \cdot 0 \\
12 \cdot 3\end{array}$ & $\begin{array}{c}4 \cdot 44 \\
9 \cdot 34-15 \cdot 4\end{array}$ & $\begin{array}{l}11 \cdot 8 \\
11 \cdot 8\end{array}$ & $\begin{array}{c}3 \cdot 60 \\
9 \cdot 25-14 \cdot 2\end{array}$ & $<0.001 \ddagger$ \\
\hline SFA (\%E) & $\begin{array}{l}\text { Mean, SD } \\
\text { Median, P25-P75 }\end{array}$ & $\begin{array}{l}16 \cdot 1 \\
15 \cdot 8\end{array}$ & $\begin{array}{c}2 \cdot 31 \\
14 \cdot 5-17 \cdot 4\end{array}$ & $\begin{array}{l}14 \cdot 3 \\
14 \cdot 2\end{array}$ & $\begin{array}{c}2 \cdot 22 \\
12 \cdot 8-15 \cdot 6\end{array}$ & $\begin{array}{l}12 \cdot 2 \\
12 \cdot 0\end{array}$ & $\begin{array}{c}1 \cdot 91 \\
11 \cdot 0-13 \cdot 4\end{array}$ & $<0.001 \ddagger$ \\
\hline MUFA (\%E) & $\begin{array}{l}\text { Mean, SD } \\
\text { Median, P25-P75 }\end{array}$ & $\begin{array}{l}12 \cdot 4 \\
12 \cdot 3\end{array}$ & $\begin{array}{c}2 \cdot 01 \\
11 \cdot 0-13 \cdot 6\end{array}$ & $\begin{array}{l}11 \cdot 4 \\
11 \cdot 3\end{array}$ & $\begin{array}{c}1 \cdot 81 \\
10 \cdot 1-12 \cdot 5\end{array}$ & $\begin{array}{l}10 \cdot 4 \\
10 \cdot 4\end{array}$ & $\begin{array}{c}1 \cdot 72 \\
9 \cdot 16-11 \cdot 4\end{array}$ & $<0.001 \ddagger$ \\
\hline PUFA (\%E) & $\begin{array}{l}\text { Mean, SD } \\
\text { Median, P25-P75 }\end{array}$ & $\begin{array}{l}3.64 \\
3.57\end{array}$ & $\begin{array}{c}0 \cdot 89 \\
3 \cdot 05-4 \cdot 19\end{array}$ & $\begin{array}{l}3.65 \\
3 \cdot 49\end{array}$ & $\begin{array}{c}0.92 \\
3.04-4.04\end{array}$ & $\begin{array}{l}3 \cdot 87 \\
3 \cdot 71\end{array}$ & $\begin{array}{c}1 \cdot 02 \\
3 \cdot 13-4 \cdot 38\end{array}$ & $0.013 \ddagger$ \\
\hline Vitamin C (mg/4184 kJ) & $\begin{array}{l}\text { Mean, SD } \\
\text { Median, P25-P75 }\end{array}$ & $\begin{array}{l}33 \cdot 3 \\
24 \cdot 42\end{array}$ & $\begin{array}{c}37 \cdot 1 \\
16 \cdot 0-37 \cdot 0\end{array}$ & $\begin{array}{l}50 \cdot 4 \\
40 \cdot 9\end{array}$ & $\begin{array}{c}50 \cdot 1 \\
27 \cdot 8-59 \cdot 5\end{array}$ & $\begin{array}{l}66.9 \\
57 \cdot 5\end{array}$ & $\begin{array}{c}43 \cdot 1 \\
43 \cdot 6-74 \cdot 8\end{array}$ & $<0.001 \S$ \\
\hline Vitamin D ( $\mu \mathrm{g} / 4184 \mathrm{~kJ})$ & $\begin{array}{l}\text { Mean, SD } \\
\text { Median, P25-P75 }\end{array}$ & $\begin{array}{l}2.49 \\
1.97\end{array}$ & $\begin{array}{c}1 \cdot 72 \\
1 \cdot 52-2 \cdot 73\end{array}$ & $\begin{array}{l}3 \cdot 13 \\
2 \cdot 45\end{array}$ & $\begin{array}{c}2 \cdot 11 \\
1 \cdot 84-3 \cdot 47\end{array}$ & $\begin{array}{l}4 \cdot 13 \\
3 \cdot 10\end{array}$ & $\begin{array}{c}2 \cdot 57 \\
2 \cdot 39-5 \cdot 00\end{array}$ & $<0.001 \S$ \\
\hline $\mathrm{Ca}(\mathrm{mg} / 4184 \mathrm{~kJ})$ & $\begin{array}{l}\text { Mean, SD } \\
\text { Median, P25-P75 }\end{array}$ & $\begin{array}{l}480 \\
459\end{array}$ & $\begin{array}{c}172 \\
337-580\end{array}$ & $\begin{array}{l}530 \\
524\end{array}$ & $\begin{array}{c}146 \\
429-621\end{array}$ & $\begin{array}{l}567 \\
554\end{array}$ & $\begin{array}{c}139 \\
468-648\end{array}$ & $<0.001 \ddagger$ \\
\hline $\mathrm{Na}(\mathrm{mg} / 4184 \mathrm{~kJ})$ & $\begin{array}{l}\text { Mean, SD } \\
\text { Median, P25-P75 }\end{array}$ & $\begin{array}{l}1432 \\
1418\end{array}$ & $\begin{array}{c}300 \\
1242-1653\end{array}$ & $\begin{array}{l}1478 \\
1489\end{array}$ & $\begin{array}{c}260 \\
1323-1672\end{array}$ & $\begin{array}{l}1432 \\
1441\end{array}$ & $\begin{array}{c}233 \\
1288-1602\end{array}$ & $0.982 \ddagger$ \\
\hline $\mathrm{Fe}(\mathrm{mg} / 4184 \mathrm{~kJ})$ & $\begin{array}{l}\text { Mean, SD } \\
\text { Median, P25-P75 }\end{array}$ & $\begin{array}{l}4 \cdot 20 \\
4 \cdot 18\end{array}$ & $\begin{array}{c}1.08 \\
3.6-4.78\end{array}$ & $\begin{array}{l}5.00 \\
4.69\end{array}$ & $\begin{array}{c}1 \cdot 51 \\
4 \cdot 06-5 \cdot 6\end{array}$ & $\begin{array}{l}5 \cdot 76 \\
5 \cdot 28\end{array}$ & $\begin{array}{c}1 \cdot 85 \\
4 \cdot 53-6 \cdot 42\end{array}$ & $<0.001 \ddagger$ \\
\hline Total HEI score & $\begin{array}{l}\text { Mean, SD } \\
\text { Median, P25-P75 }\end{array}$ & $\begin{array}{l}45 \cdot 7 \\
46 \cdot 6\end{array}$ & $\begin{array}{c}3 \cdot 48 \\
43 \cdot 6-48 \cdot 7\end{array}$ & $\begin{array}{l}60 \cdot 3 \\
60 \cdot 2\end{array}$ & $\begin{array}{c}5 \cdot 25 \\
55 \cdot 8-64 \cdot 5\end{array}$ & $\begin{array}{l}73 \cdot 0 \\
72 \cdot 2\end{array}$ & $\begin{array}{c}2 \cdot 56 \\
70 \cdot 9-74 \cdot 3\end{array}$ & $<0.001 \ddagger$ \\
\hline Total Fruit HEI score & $\begin{array}{l}\text { Mean, SD } \\
\text { Median, P25-P75 }\end{array}$ & $\begin{array}{l}1.32 \\
0.99\end{array}$ & $\begin{array}{c}1 \cdot 30 \\
0.00-2 \cdot 01\end{array}$ & $\begin{array}{l}3 \cdot 19 \\
3.22\end{array}$ & $\begin{array}{c}1 \cdot 62 \\
1 \cdot 75-5.00\end{array}$ & $\begin{array}{l}4.46 \\
5 \cdot 00\end{array}$ & $\begin{array}{c}0.89 \\
4 \cdot 16-5 \cdot 00\end{array}$ & $<0.001 \ddagger$ \\
\hline Whole Fruit HEI score & $\begin{array}{l}\text { Mean, SD } \\
\text { Median, P25-P75 }\end{array}$ & $\begin{array}{l}1.37 \\
0.72\end{array}$ & $\begin{array}{c}1 \cdot 56 \\
0 \cdot 00-2 \cdot 26\end{array}$ & $\begin{array}{l}3 \cdot 51 \\
4 \cdot 21\end{array}$ & $\begin{array}{c}1 \cdot 74 \\
1 \cdot 90-5 \cdot 00\end{array}$ & $\begin{array}{l}4 \cdot 83 \\
5 \cdot 00\end{array}$ & $\begin{array}{c}0.55 \\
5 \cdot 00-5.00\end{array}$ & $<0.001 \ddagger$ \\
\hline Total Vegetable HEI score & $\begin{array}{l}\text { Mean, SD } \\
\text { Median, P25-P75 }\end{array}$ & $\begin{array}{l}2 \cdot 26 \\
2 \cdot 16\end{array}$ & $\begin{array}{c}1.03 \\
1.52-2 \cdot 95\end{array}$ & $\begin{array}{l}3.03 \\
3.05\end{array}$ & $\begin{array}{c}1 \cdot 22 \\
2 \cdot 14-4 \cdot 1\end{array}$ & $\begin{array}{l}3 \cdot 84 \\
4 \cdot 11\end{array}$ & $\begin{array}{c}1 \cdot 04 \\
3 \cdot 12-4 \cdot 86\end{array}$ & $<0.001 \ddagger$ \\
\hline DGaOVaL HEI score & $\begin{array}{l}\text { Mean, SD } \\
\text { Median, P25-P75 }\end{array}$ & $\begin{array}{l}0.28 \\
0.02\end{array}$ & $\begin{array}{c}0.47 \\
0.00-0.35\end{array}$ & $\begin{array}{l}0.66 \\
0.29\end{array}$ & $\begin{array}{c}0.86 \\
0.01-0.97\end{array}$ & $\begin{array}{l}1 \cdot 78 \\
1 \cdot 47\end{array}$ & $\begin{array}{c}1 \cdot 46 \\
0 \cdot 47-2 \cdot 79\end{array}$ & $<0.001 \S$ \\
\hline Total Grain HEl score & $\begin{array}{l}\text { Mean, SD } \\
\text { Median, P25-P75 }\end{array}$ & $\begin{array}{l}3 \cdot 82 \\
3 \cdot 88\end{array}$ & $\begin{array}{c}0.99 \\
3 \cdot 22-5 \cdot 00\end{array}$ & $\begin{array}{l}4 \cdot 34 \\
4 \cdot 66\end{array}$ & $\begin{array}{c}0 \cdot 77 \\
3 \cdot 87-5 \cdot 00\end{array}$ & $\begin{array}{l}4 \cdot 65 \\
5 \cdot 00\end{array}$ & $\begin{array}{c}0.57 \\
4 \cdot 33-5 \cdot 00\end{array}$ & $<0.001 \ddagger$ \\
\hline
\end{tabular}


Children with higher total HEI scores had a more expensive diet, and those spending the most money on food consumed a healthier diet. This is in line with other studies showing that healthy diets cost more ${ }^{(16-21,24)}$. The magnitude of the difference in cost between healthy and less-healthy diets might seem rather small $(€ 0 \cdot 34 / 4184 \mathrm{~kJ}$ ( $1000 \mathrm{kcal})$ ), but it corresponds to approximately $€ 1000 /$ year for a family of four.

There are most likely several reasons why healthier diets are more expensive. One reason is that energydense foods may be cheaper, as measured by cost per kilojoule $^{(32)}$. In our study, energy density decreased when food expenses and the healthiness of the diet increased, as has also been shown by others ${ }^{(16,44)}$. Because energy density can serve as a marker for dietary quality ${ }^{(31)}$ - the lower the energy density, the better the dietary quality - it makes sense that those consuming an energy-dense diet scored low on the HEI. Those with high scores on the HEI spent approximately $25 \%$ of their food budget on fruit and vegetables, food groups characterized by their low energy density, whereas the low HEI-score group spent $25 \%$ of the food budget on discretionary calories, for which energy density is high. Another explanation for why energy density is associated with diet cost is that one must eat more food to reach energy equilibrium if foods with low energy density are chosen ${ }^{(45)}$. Although food prices differ among and within food groups, almost all foods cost money, and increased food intake when eating low energy-dense diets will thereby increase food costs.

Another reason why healthier diets are more expensive might be due to price differences among healthy and lesshealthy products within food groups. However, healthier options within a food group are not necessarily more expensive. Wholegrain products and healthier fat products are, in fact, cheaper per gram compared with their respective food groups as a whole. Healthy dairy products, on the other hand, are about twice as expensive as the total dairy group, and the average price for healthier meat options was about $€ 1$ more expensive per kilogram. This indicates that within some food groups, it is possible to choose healthier alternatives without increasing food costs, whereas in other food groups, food costs are likely to increase if healthier alternatives are chosen.

Yet another reason why healthier diets are more expensive may be due to the amount of intake within more expensive food groups, if these coincide with healthier food groups. Multiple regression analysis showed that fish, meat, fruit and ready meals accounted for more than half of the differences in total cost. With the exception of fruit, these food groups represented those with the highest cost per gram. They also represented food groups in which relative intakes increased the most among cost quintiles. This increased intake in approximately the same food groups was also found in other studies when intake was stratified by total dietary $\operatorname{cost}^{(23,44)}$. High intakes of fish and fruit are consistent 


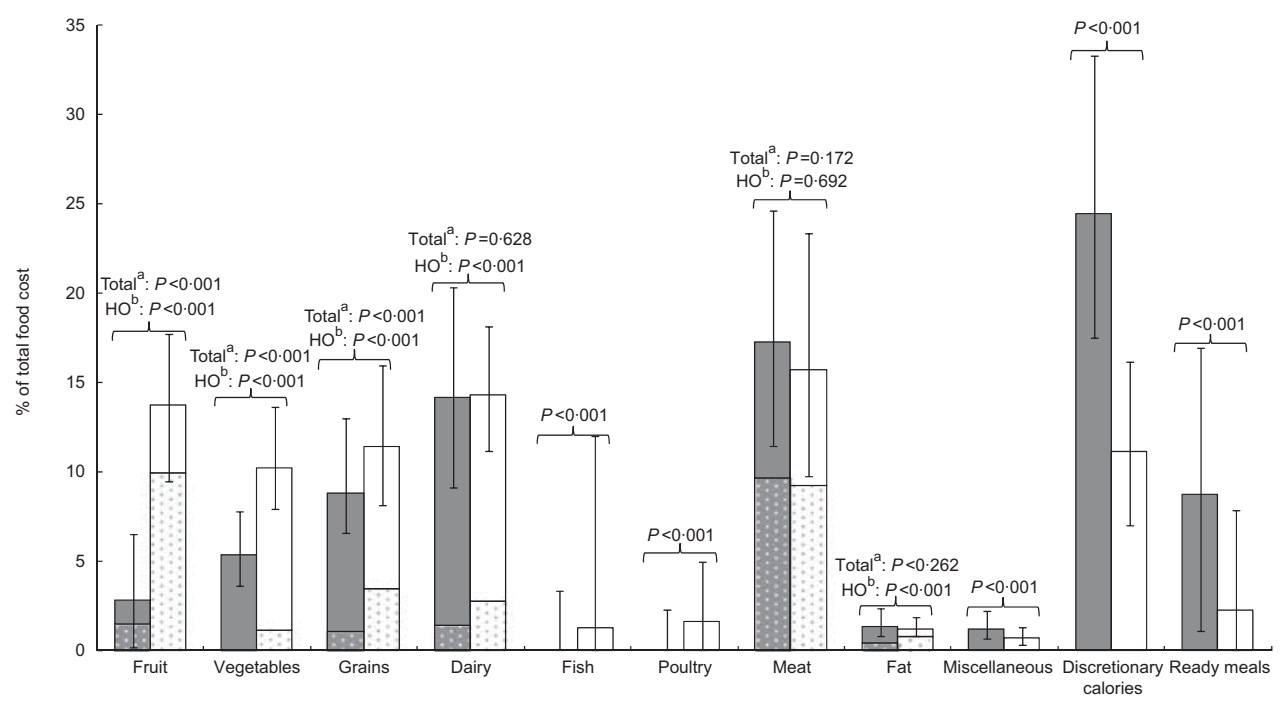

Fig. 1 Comparison of cost per food group, subdivided into the healthier option $(\mathrm{HO})$ within the food group where applicable (回, 四), as median percentage of total food cost between the group with low 2005 Healthy Eating Index (HEI) score $(\mathrm{HEI}<50, n 221 ; \square)$ and the group with high $\mathrm{HEI}$ score ( $\mathrm{HEI}>70, n 202 ; \square)$, with 25 th and 75 th percentile values represented by error bars. ${ }^{a} P$ value for the whole food group including part from the $\mathrm{HO} ;{ }^{b} \mathrm{P}$ value for the $\mathrm{HO}$ part of the food group

with healthy eating, but ready meals and meat are not generally considered to be healthy ${ }^{(46)}$. However, more than half of the meat consumption in the highest cost quintile consisted of healthier options, such as lean meat and low-fat products. The healthiness of ready meals varies greatly depending on product type, and meals at restaurants are likely to be healthier than fast-food meals ${ }^{(47)}$. In Sweden, it is generally more expensive to dine at restaurants than to buy fast foods, and the higher average cost for ready meals in the highest cost quintile could be due to a higher proportion of restaurant meals. Therefore, total food cost will increase if healthier food choices lead to higher intake from food groups in which average prices are high, even more so if healthier selections within food groups have a higher price than the group average.

A final reason why healthier diets are more expensive is that healthy diets often include a greater variety of foods ${ }^{(48)}$. In our study, the highest cost quintile consumed the largest number of unique food items, as did the high HEI-score group. On a food group level, fruit, vegetables, fish, meat (healthier options) and ready meals accounted for the majority of the difference in variety. These food groups are also those in which relative intake and cost per gram increases the most with rising food costs. This implies that a larger intake within food groups will comprise greater variety, perhaps because it might not be satisfying to eat the same food repeatedly. It also implies that it might not be possible to find alternative foods within the same food group at the same price; therefore, dietary costs would rise with increasing dietary variety.

A possible consequence of higher dietary costs when eating healthily is that low-SEP groups will find it too expensive to adhere to a healthy diet. In the present study, we found a small but significant indication that children whose parents had lower educational and occupational levels consumed a cheaper and less-healthy diet. The relationship between dietary quality and SEP is in line with several other studies based on both Swedish and international data ${ }^{(2-7,49)}$. There are, however, few studies examining the relationship between actual dietary costs and SEP. Waterlander et al. found no difference in actual food costs among income levels in adults; however, they suggested that the lack of significance could be due to the small number of participants in the lowincome groups ${ }^{(21)}$. On the other hand, low-SEP groups have been found to spend less money on food ${ }^{(50,51)}$ and to have less-healthy food purchasing behaviour ${ }^{(50,52,53)}$, indicating the likelihood of lower actual dietary costs with lower SEP. This implies that one of the reasons for the less-healthy dietary pattern associated with low SEP is the cost of healthy eating.

Some limitations of our study are worth noting. Although we excluded obvious dietary under-reporters, our cut-off point at EI:BMR $=1.06$ is likely to have allowed some under-reporters to be included. Because under-reporting is biased towards unhealthy foods ${ }^{(54)}$, it is possible that the high HEI scores were overestimated. This is indicated by the differences in food intake levels among HEI groups. However, it is unclear to what extent total food costs might have been affected by selective under-reporting; there was a significant difference in food intake level between the highest and lowest cost quintiles, but it is likely that the energy adjustment decreased the effect of the under-reporters.

Disposable income is likely a more important determinant with regard to food budget choice than SEP markers such as education and occupation. Although 
Table 4 Characteristics of participants and their food intake according to daily food costs. Data are presented as mean and standard deviation for normally distributed variables or median and 25th-75th percentile where data are skewed

\begin{tabular}{|c|c|c|c|c|c|c|c|c|c|c|c|c|}
\hline & & \multicolumn{10}{|c|}{ Quintile of daily food consumption cost (€/4184 kJ) } & \multirow[b]{3}{*}{$P$ value } \\
\hline & & \multicolumn{2}{|c|}{1 (lowest; $n$ 427) } & \multicolumn{2}{|c|}{$2(n 438)$} & \multicolumn{2}{|c|}{$3(n 446)$} & \multicolumn{2}{|c|}{$4(n 440)$} & \multicolumn{2}{|c|}{5 (highest; $n$ 409) } & \\
\hline & & $\begin{array}{l}\text { Mean or } \\
\text { Median }\end{array}$ & $\begin{array}{c}\text { SD or } \\
\text { P25-P75 }\end{array}$ & $\begin{array}{l}\text { Mean or } \\
\text { Median }\end{array}$ & $\begin{array}{c}\text { SD or } \\
\text { P25-P75 }\end{array}$ & $\begin{array}{l}\text { Mean or } \\
\text { Median }\end{array}$ & $\begin{array}{c}\text { SD or } \\
\text { P25-P75 }\end{array}$ & $\begin{array}{l}\text { Mean or } \\
\text { Median }\end{array}$ & $\begin{array}{c}\text { SD or } \\
\text { P25-P75 }\end{array}$ & $\begin{array}{l}\text { Mean or } \\
\text { Median }\end{array}$ & $\begin{array}{c}\text { SD or } \\
\text { P25-P75 }\end{array}$ & \\
\hline Total cost (€/4184 kJ) & Mean, SD & $1 \cdot 84$ & $0 \cdot 14$ & $2 \cdot 13$ & 0.06 & $2 \cdot 33$ & 0.06 & $2 \cdot 57$ & 0.09 & $3 \cdot 08$ & 0.33 & $<0.001 \ddagger$ \\
\hline Total HEI score & Mean, SD & $57 \cdot 5$ & $7 \cdot 60$ & $58 \cdot 5$ & $7 \cdot 80$ & $60 \cdot 3$ & $7 \cdot 59$ & $61 \cdot 4$ & $7 \cdot 58$ & $62 \cdot 3$ & $7 \cdot 45$ & $<0 \cdot 001 \ddagger$ \\
\hline Age (years) & & $8 \cdot 67$ & $2 \cdot 97$ & $8 \cdot 67$ & $2 \cdot 93$ & $8 \cdot 40$ & $2 \cdot 84$ & $8 \cdot 49$ & $2 \cdot 93$ & $8 \cdot 34$ & $2 \cdot 86$ & $0 \cdot 325 \ddagger$ \\
\hline Boys (\%) & & $54 \cdot 6$ & & $56 \cdot 2$ & & $52 \cdot 5$ & & $48 \cdot 0$ & & $43 \cdot 5$ & & $0.001 \S$ \\
\hline ISO-BMI'> 25 kg/m² (\%)† & & $18 \cdot 8$ & & $13 \cdot 0$ & & $15 \cdot 6$ & & $16 \cdot 1$ & & $16 \cdot 0$ & & $0.295 \S$ \\
\hline Food intake level (El:BMR) & Mean, SD & $1 \cdot 60$ & 0.35 & $1 \cdot 61$ & 0.33 & $1 \cdot 60$ & 0.31 & $1 \cdot 60$ & 0.31 & $1 \cdot 55$ & 0.29 & $0.079 \ddagger$ \\
\hline Energy density $(\mathrm{kJ} / \mathrm{g})$ & Mean, SD & 4.53 & $0 \cdot 81$ & $4 \cdot 34$ & 0.66 & $4 \cdot 20$ & 0.66 & $4 \cdot 18$ & 0.63 & $4 \cdot 05$ & 0.56 & $<0.001 \ddagger$ \\
\hline Energy intake (kJ) & Mean, SD & 7668 & 1889 & 7670 & 1563 & 7594 & 1750 & 7588 & 1732 & 7247 & 1508 & $0.001 \ddagger$ \\
\hline Variation (no. of unique items) & Mean, SD & $46 \cdot 0$ & $10 \cdot 2$ & $50 \cdot 5$ & $10 \cdot 7$ & 52.5 & $10 \cdot 4$ & 53.3 & $10 \cdot 5$ & $54 \cdot 2$ & $9 \cdot 95$ & $<0.001 \ddagger$ \\
\hline Fruit $(€ / 100 \mathrm{~g})$ & Mean, SD & $0 \cdot 20$ & $0 \cdot 11$ & 0.21 & $0 \cdot 10$ & 0.22 & 0.09 & 0.22 & 0.09 & 0.23 & $0 \cdot 10$ & $<0.001 \ddagger$ \\
\hline Whole fruit $(€ / 100 \mathrm{~g})$ & $\begin{array}{l}\text { Median, } \\
\text { P25-P75 }\end{array}$ & $0 \cdot 20$ & $0 \cdot 18-0 \cdot 22$ & $0 \cdot 20$ & $0 \cdot 18-0 \cdot 23$ & $0 \cdot 21$ & $0 \cdot 19-0 \cdot 24$ & $0 \cdot 20$ & $0 \cdot 19-0 \cdot 24$ & $0 \cdot 21$ & $0 \cdot 19-0 \cdot 27$ & $<0.001 \S$ \\
\hline Vegetables (€/100 g) & Mean, SD & $0 \cdot 17$ & 0.08 & $0 \cdot 19$ & 0.08 & $0 \cdot 20$ & 0.09 & $0 \cdot 20$ & 0.08 & 0.22 & 0.09 & $<0.001 \ddagger$ \\
\hline DGaOVaL (€/100 g) & $\begin{array}{l}\text { Median, } \\
\text { P25-P75 }\end{array}$ & $0 \cdot 11$ & $0 \cdot 00-0 \cdot 17$ & $0 \cdot 11$ & $0 \cdot 11-0 \cdot 18$ & $0 \cdot 11$ & $0 \cdot 11-0 \cdot 23$ & $0 \cdot 11$ & $0 \cdot 11-0 \cdot 28$ & $0 \cdot 11$ & $0 \cdot 11-0 \cdot 22$ & $<0 \cdot 001 \S$ \\
\hline Grains $(€ / 100 \mathrm{~g})$ & Mean, SD & 0.35 & 0.09 & 0.36 & 0.08 & 0.37 & 0.08 & 0.37 & 0.09 & 0.36 & 0.07 & $<0.001 \ddagger$ \\
\hline Whole grains $(€ / 100 \mathrm{~g})$ & Mean, SD & 0.34 & $0 \cdot 17$ & 0.35 & $0 \cdot 17$ & 0.35 & $0 \cdot 16$ & 0.35 & $0 \cdot 17$ & 0.35 & $0 \cdot 15$ & $0.978 \ddagger$ \\
\hline Dairy $(€ / 100 \mathrm{~g})$ & Mean, SD & $0 \cdot 12$ & 0.05 & $0 \cdot 13$ & 0.04 & $0 \cdot 12$ & 0.04 & $0 \cdot 14$ & 0.06 & $0 \cdot 13$ & 0.04 & $<0.001 \ddagger$ \\
\hline Dairy, HO (€/100 g) & $\begin{array}{l}\text { Median, } \\
\text { P25-P75 }\end{array}$ & $0 \cdot 21$ & $0 \cdot 14-0 \cdot 40$ & $0 \cdot 20$ & $0 \cdot 14-0 \cdot 40$ & $0 \cdot 27$ & $0 \cdot 15-0 \cdot 40$ & $0 \cdot 22$ & $0 \cdot 14-0 \cdot 40$ & $0 \cdot 21$ & $0 \cdot 13-0 \cdot 40$ & $0 \cdot 180 \S$ \\
\hline Fish $(€ / 100 \mathrm{~g})$ & $\begin{array}{l}\text { Median, } \\
\text { P25-P75 }\end{array}$ & $0 \cdot 00$ & $0 \cdot 00-1 \cdot 09$ & $0 \cdot 00$ & $0 \cdot 00-1 \cdot 29$ & $0 \cdot 00$ & $0 \cdot 00-1 \cdot 31$ & $1 \cdot 09$ & $0 \cdot 00-1 \cdot 31$ & $1 \cdot 31$ & $0 \cdot 00-1 \cdot 36$ & $<0.001 \S$ \\
\hline Poultry $(€ / 100 \mathrm{~g})$ & $\begin{array}{l}\text { Median, } \\
\text { P25-P75 }\end{array}$ & $0 \cdot 00$ & $0 \cdot 00-0 \cdot 32$ & $0 \cdot 00$ & $0 \cdot 00-0 \cdot 32$ & $0 \cdot 32$ & $0 \cdot 00-0 \cdot 37$ & $0 \cdot 32$ & $0 \cdot 00-0.52$ & $0 \cdot 32$ & $0 \cdot 00-0 \cdot 75$ & $<0.001 \S$ \\
\hline Meat $(€ / 100 \mathrm{~g})$ & Mean, SD & 0.72 & $0 \cdot 19$ & 0.75 & $0 \cdot 17$ & 0.78 & $0 \cdot 19$ & 0.82 & $0 \cdot 20$ & 0.87 & 0.24 & $<0.001 \ddagger$ \\
\hline Meat, $\mathrm{HO}(€ / 100 \mathrm{~g})$ & Mean, SD & $0 \cdot 84$ & 0.33 & $0 \cdot 86$ & $0 \cdot 27$ & $0 \cdot 89$ & $0 \cdot 27$ & $0 \cdot 91$ & 0.29 & 0.93 & 0.33 & $<0.001 \ddagger$ \\
\hline Fat $(€ / 100 \mathrm{~g})$ & Mean, SD & 0.45 & $0 \cdot 15$ & 0.46 & $0 \cdot 16$ & 0.47 & $0 \cdot 16$ & 0.46 & $0 \cdot 17$ & 0.48 & $0 \cdot 16$ & $0 \cdot 115 \ddagger$ \\
\hline Fat, $\mathrm{HO}(€ / 100 \mathrm{~g})$ & Mean, SD & 0.33 & 0.14 & 0.33 & $0 \cdot 16$ & 0.34 & $0 \cdot 16$ & 0.34 & $0 \cdot 15$ & 0.34 & $0 \cdot 16$ & $0.988 \ddagger$ \\
\hline Miscellaneous (€/100 g) & $\begin{array}{l}\text { Median, } \\
\text { P25-P75 }\end{array}$ & 0.03 & $0.01-0.09$ & 0.03 & $0 \cdot 01-0.07$ & 0.03 & $0.01-0.07$ & 0.03 & $0 \cdot 01-0.06$ & 0.03 & $0 \cdot 01-0.07$ & $0 \cdot 101 \S$ \\
\hline Discretionary calories $(€ / 100 \mathrm{~g})$ & Mean, SD & 0.29 & $0 \cdot 13$ & $0 \cdot 31$ & $0 \cdot 15$ & 0.33 & $0 \cdot 18$ & $0 \cdot 32$ & $0 \cdot 15$ & 0.36 & $0 \cdot 21$ & $<0 \cdot 001 \ddagger$ \\
\hline Ready meals $(€ / 100 \mathrm{~g})$ & $\begin{array}{l}\text { Median, } \\
\text { P25-P75 }\end{array}$ & $0 \cdot 45$ & $0 \cdot 00-0 \cdot 70$ & $0 \cdot 46$ & $0 \cdot 00-0 \cdot 89$ & 0.54 & $0 \cdot 00-0 \cdot 89$ & $0 \cdot 64$ & $0 \cdot 00-1 \cdot 09$ & $0 \cdot 88$ & $0 \cdot 44-1 \cdot 42$ & $<0 \cdot 001 \S$ \\
\hline All foods $(€ / 100 \mathrm{~g})$ & Mean, SD & 0.34 & 0.07 & 0.36 & 0.07 & 0.38 & 0.07 & 0.41 & 0.09 & 0.43 & 0.07 & $<0.001 \ddagger$ \\
\hline Fruit $(\mathrm{g} / 4184 \mathrm{~kJ}$ per $\mathrm{d})$ & $\begin{array}{l}\text { Median, } \\
\text { P25-P75 }\end{array}$ & $52 \cdot 0$ & $20 \cdot 9-92 \cdot 7$ & $68 \cdot 3$ & $32 \cdot 8-118$ & $89 \cdot 5$ & $47 \cdot 5-143$ & $100 \cdot 8$ & $47 \cdot 7-153$ & $114 \cdot 6$ & $63 \cdot 7-178$ & $<0.001 \S$ \\
\hline Whole fruit (g/4184 kJ per d) & $\begin{array}{l}\text { Median, } \\
\text { P25-P75 }\end{array}$ & $34 \cdot 2$ & $13 \cdot 1-64 \cdot 1$ & $37 \cdot 7$ & $15 \cdot 8-69 \cdot 3$ & $52 \cdot 6$ & $24 \cdot 3-82 \cdot 6$ & $56 \cdot 4$ & $26 \cdot 4-93 \cdot 2$ & $66 \cdot 2$ & $38 \cdot 4-114$ & $<0.001 \S$ \\
\hline Vegetables (g/4184 kJ per d) & Mean, SD & $82 \cdot 4$ & $43 \cdot 6$ & $85 \cdot 3$ & $41 \cdot 7$ & $90 \cdot 1$ & $42 \cdot 5$ & $99 \cdot 4$ & $44 \cdot 7$ & $104 \cdot 7$ & $49 \cdot 6$ & $<0 \cdot 001 \ddagger$ \\
\hline DGaOVaL (g/4184 kJ per d) & $\begin{array}{l}\text { Median, } \\
\text { P25-P75 }\end{array}$ & 0.96 & $0 \cdot 00-7 \cdot 83$ & $3 \cdot 39$ & $0 \cdot 06-9 \cdot 83$ & $3 \cdot 72$ & $0 \cdot 11-11 \cdot 12$ & 3.95 & $0 \cdot 10-12 \cdot 84$ & $4 \cdot 28$ & $0 \cdot 52-14 \cdot 20$ & $<0 \cdot 001 \S$ \\
\hline Grains (g/4184 kJ per d) & $\begin{array}{l}\text { Median, } \\
\text { P25-P75 }\end{array}$ & $96 \cdot 0$ & $72 \cdot 2-131$ & $96 \cdot 8$ & $76 \cdot 5-129$ & $91 \cdot 9$ & $74 \cdot 5-118$ & $91 \cdot 6$ & $73 \cdot 6-115$ & $89 \cdot 0$ & $69 \cdot 7-116$ & $0.005 \S$ \\
\hline
\end{tabular}




\begin{tabular}{|c|c|c|c|c|c|c|c|c|c|c|c|c|}
\hline & & \multicolumn{10}{|c|}{ Quintile of daily food consumption cost ( $€ / 4184 \mathrm{~kJ})$} & \multirow[b]{3}{*}{$P$ value } \\
\hline & & \multicolumn{2}{|c|}{1 (lowest; $n$ 427) } & \multicolumn{2}{|c|}{$2(n 438)$} & \multicolumn{2}{|c|}{$3(n 446)$} & \multicolumn{2}{|c|}{$4(n 440)$} & \multicolumn{2}{|c|}{5 (highest; $n$ 409) } & \\
\hline & & $\begin{array}{l}\text { Mean or } \\
\text { Median }\end{array}$ & $\begin{array}{c}\text { SD or } \\
\text { P25-P75 }\end{array}$ & $\begin{array}{l}\text { Mean or } \\
\text { Median }\end{array}$ & $\begin{array}{c}\text { SD or } \\
\text { P25-P75 }\end{array}$ & $\begin{array}{l}\text { Mean or } \\
\text { Median }\end{array}$ & $\begin{array}{c}\text { SD or } \\
\text { P25-P75 }\end{array}$ & $\begin{array}{l}\text { Mean or } \\
\text { Median }\end{array}$ & $\begin{array}{c}\text { SD or } \\
\text { P25-P75 }\end{array}$ & $\begin{array}{l}\text { Mean or } \\
\text { Median }\end{array}$ & $\begin{array}{c}\text { SD or } \\
\text { P25-P75 }\end{array}$ & \\
\hline Whole grains $(g / 4184 \mathrm{~kJ}$ per $\mathrm{d})$ & $\begin{array}{l}\text { Median, } \\
\text { P25-P75 }\end{array}$ & $13 \cdot 0$ & $4 \cdot 64-28 \cdot 2$ & $12 \cdot 6$ & $5 \cdot 15-29 \cdot 1$ & $14 \cdot 8$ & $5 \cdot 85-28 \cdot 3$ & $12 \cdot 8$ & $4 \cdot 82-25 \cdot 0$ & $14 \cdot 8$ & $6 \cdot 23-27 \cdot 2$ & $0.498 \S$ \\
\hline Dairy $(g / 4184 \mathrm{~kJ}$ per d) & Mean, SD & 323 & 132 & 310 & 131 & 311 & 119 & 286 & $\begin{array}{c}124 \\
3.82-53.7\end{array}$ & 286 & $\begin{array}{c}124 \\
16-51 \cdot 7\end{array}$ & $<0.001 \mp$ \\
\hline Dairy, HO (g/4184 kJ per d) & $\begin{array}{l}\text { Median, } \\
\text { P25-P75 }\end{array}$ & $20 \cdot 0$ & $4 \cdot 25-52 \cdot 1$ & $18 \cdot 4$ & $4 \cdot 65-47 \cdot 1$ & $14 \cdot 9$ & $3 \cdot 50-46 \cdot 6$ & $19 \cdot 1$ & $3 \cdot 82-53 \cdot 7$ & $21 \cdot 0$ & $4 \cdot 46-51 \cdot 7$ & $0 \cdot 474 \S$ \\
\hline Fish (g/4184 kJ per d) & $\begin{array}{l}\text { Median, } \\
\text { P25-P75 }\end{array}$ & $0 \cdot 00$ & $0 \cdot 00-1 \cdot 76$ & 0.00 & $0 \cdot 00-6 \cdot 47$ & 0.00 & $0.00-9.56$ & $2 \cdot 82$ & $0 \cdot 00-13 \cdot 5$ & $10 \cdot 8$ & $0 \cdot 00-22 \cdot 8$ & $<0.001 \S$ \\
\hline Poultry (g/4184 kJ per d) & $\begin{array}{l}\text { Median, } \\
\text { P25-P75 }\end{array}$ & $0 \cdot 00$ & $0 \cdot 00-9 \cdot 17$ & 0.00 & $0 \cdot 00-11 \cdot 7$ & $2 \cdot 20$ & $0 \cdot 00-12 \cdot 2$ & $2 \cdot 33$ & $0 \cdot 00-13 \cdot 0$ & $2 \cdot 48$ & $0 \cdot 00-15 \cdot 3$ & $<0.001 \S$ \\
\hline Meat $(\mathrm{g} / 4184 \mathrm{~kJ}$ per $\mathrm{d})$ & Mean, SD & $41 \cdot 3$ & $19 \cdot 4$ & $45 \cdot 6$ & $20 \cdot 3$ & $49 \cdot 1$ & $20 \cdot 5$ & $52 \cdot 5$ & $24 \cdot 0$ & $56 \cdot 8$ & $27 \cdot 8$ & $<0.001 \ddagger$ \\
\hline Meat, HO (g/4184 kJ per d) & $\begin{array}{l}\text { Median, } \\
\text { P25-P75 }\end{array}$ & $15 \cdot 3$ & $8 \cdot 21-23 \cdot 9$ & $19 \cdot 1$ & $10 \cdot 6-30 \cdot 8$ & $21 \cdot 6$ & $12 \cdot 0-32 \cdot 4$ & $23 \cdot 2$ & $12 \cdot 9-35 \cdot 3$ & $27 \cdot 0$ & $15 \cdot 6-39 \cdot 2$ & $<0.001 \S$ \\
\hline Fat $(g / 4184 \mathrm{~kJ}$ per $\mathrm{d})$ & $\begin{array}{l}\text { Median, } \\
\text { P25-P75 }\end{array}$ & $7 \cdot 35$ & $4 \cdot 94-11 \cdot 1$ & $7 \cdot 14$ & $4 \cdot 87-10 \cdot 1$ & $7 \cdot 08$ & $4 \cdot 64-10 \cdot 4$ & $6 \cdot 65$ & $3 \cdot 97-9 \cdot 72$ & $6 \cdot 59$ & $4 \cdot 19-10 \cdot 2$ & $0.013 \S$ \\
\hline Fat, HO (g/4184 kJ/day) & $\begin{array}{l}\text { Median, } \\
\text { P25-P75 }\end{array}$ & $4 \cdot 53$ & $2 \cdot 13-8 \cdot 27$ & $4 \cdot 50$ & $1 \cdot 47-7 \cdot 84$ & $4 \cdot 37$ & $1 \cdot 70-7 \cdot 33$ & $3 \cdot 94$ & $1 \cdot 64-7 \cdot 24$ & $4 \cdot 03$ & $1 \cdot 55-6 \cdot 68$ & $0.063 \S$ \\
\hline Miscellaneous (g/4184 kJ per d) & $\begin{array}{l}\text { Median, } \\
\text { P25-P75 }\end{array}$ & $78 \cdot 1$ & $31 \cdot 2-148$ & $92 \cdot 2$ & $42 \cdot 8-174$ & $98 \cdot 4$ & $41 \cdot 8-162$ & $92 \cdot 3$ & $42 \cdot 8-171$ & 106 & $45 \cdot 8-186$ & $0.001 \S$ \\
\hline $\begin{array}{l}\text { Discretionary calories }(\mathrm{g} / 4184 \mathrm{~kJ} \\
\text { per d) }\end{array}$ & $\begin{array}{l}\text { Median, } \\
\text { P25-P75 }\end{array}$ & 177 & $114-252$ & 178 & $118-262$ & 181 & $118-249$ & 177 & $115-262$ & 171 & $114-235$ & $0.523 \S$ \\
\hline Ready meals (g/4184kJ per d) & $\begin{array}{l}\text { Median, } \\
\text { P25-P75 }\end{array}$ & $11 \cdot 7$ & $0 \cdot 00-25 \cdot 2$ & $15 \cdot 1$ & $0 \cdot 00-30 \cdot 8$ & $18 \cdot 7$ & $0 \cdot 00-36 \cdot 6$ & $20 \cdot 4$ & $0 \cdot 00-40 \cdot 9$ & $24 \cdot 8$ & $7 \cdot 69-48 \cdot 6$ & $<0.001 \S$ \\
\hline All foods (g/4184 kJ per d) & Mean, SD & 956 & 168 & 988 & 154 & 1016 & 162 & 1026 & 165 & 1068 & 174 & $<0.001 \ddagger$ \\
\hline
\end{tabular}

HEI, 2005 Healthy Eating Index; ISO-BMI, age-adjusted BMI; El, energy intake; DGaOVaL, dark green and orange vegetables and legumes; HO, healthier option. $4184 \mathrm{~kJ}=1000 \mathrm{kcal}$.

+Q1, $n$ 373; Q2, $n$ 399; Q3, $n$ 397; Q4, $n$ 386; Q5, $n 371$.

$\ddagger P$ for difference between groups as calculated by ANOVA.

$\S P$ for difference between groups as calculated by $\chi^{2}$ (sex and ISO-BMI) or Kruskal-Wallis test. 
these SEP markers can be seen as reflecting income, recent studies have shown that financial situation, rather than education or occupation, is associated with a higher fruit and vegetable intake ${ }^{(5,8,55)}$. Knowledge of disposable income and/or the current financial situation of children's families might have strengthened the weak relationship between SEP groups and diet cost in our study.

Although we put much effort into verifying the accuracy of food price information, there were some shortcomings: the lack of details about the foods consumed made it necessary for us to use the average price of each food instead of using actual prices. It is likely that lowSEP families use discounts and low-cost foods to reduce food costs ${ }^{(50)}$ to a larger extent, which would increase differences in total food costs compared with high-SEP families. Furthermore, food prices were collected 7 years after the diet survey was conducted. During these years, prices might have changed both within and among food groups ${ }^{(56)}$, which in turn could have affected dietary intake, because cost is one determinant of food purchase $^{(57)}$. It is also likely that the ready meals food group was underestimated, as all meals were considered homecooked unless clearly stated otherwise. This could change the impact of ready meals on total food costs, but because the food group comprises meals bought in grocery stores, meals from restaurants and fast food, it is unclear how the underestimation would have affected food costs.

It is also important to consider that all of the participants were children. In Sweden, all school children are provided free school lunches and a majority of 4-yearolds are enrolled in day-care, where all meals are free. However, costs for these meals were calculated as if they were home-cooked. Both school and day-care meals are usually planned by nutritionally trained managers (although often with budget restraints). Hence, dietary intakes might be healthier in these settings than diets eaten within a family setting.

The strength of our study is that dietary data were based on a large number of nationally representative children using an open-survey technique (food diaries) instead of a predefined technique, such as an FFQ. When considering food costs, it is important to investigate food intake in as much detail as possible.

\section{Conclusion}

Healthy eating was associated with higher dietary cost in Swedish children. Important reasons for this are: (i) differences in food prices, in which healthier options such as fish and lean meats were more expensive; (ii) increased intakes of less energy-dense foods; and (iii) a higher cost when consuming a more varied diet. Because higher dietary costs are likely to be a barrier for low-income groups with regard to eating healthily, as indicated in our study, this result poses challenges for public health policies, 
Table 6 Socio-economic position in relation to total dietary cost and total HEI score

\begin{tabular}{|c|c|c|c|c|c|c|c|c|}
\hline & \multicolumn{4}{|c|}{ Cost $(€ / 4184 \mathrm{~kJ})$} & \multicolumn{4}{|c|}{ Total HEI score } \\
\hline & Mean & $95 \% \mathrm{Cl}$ & Adjusted meant & $95 \% \mathrm{Cl}$ & Mean & $95 \% \mathrm{Cl}$ & Adjusted mean & $95 \% \mathrm{Cl}$ \\
\hline \multicolumn{9}{|l|}{ Parental education } \\
\hline Primary ( $n 73)$ & $2 \cdot 25$ & $2 \cdot 14,2 \cdot 35$ & $2 \cdot 29$ & $2 \cdot 18,2 \cdot 39$ & $56 \cdot 41$ & $54 \cdot 75,58 \cdot 07$ & $57 \cdot 58$ & $55 \cdot 85,59 \cdot 28$ \\
\hline Secondary $\leq 2$ years $(n 479)$ & $2 \cdot 35$ & $2 \cdot 31,2 \cdot 39$ & $2 \cdot 36$ & $2 \cdot 32,2 \cdot 40$ & $59 \cdot 05$ & $58 \cdot 34,59 \cdot 77$ & $59 \cdot 46$ & $58 \cdot 78,60 \cdot 11$ \\
\hline Secondary $\geq 3$ years $(n 542)$ & $2 \cdot 38$ & $2 \cdot 34,2 \cdot 42$ & $2 \cdot 38$ & $2 \cdot 34,2 \cdot 42$ & $60 \cdot 00$ & $59 \cdot 35,60 \cdot 65$ & $59 \cdot 83$ & $59 \cdot 12,60 \cdot 46$ \\
\hline University ( $n$ 1023) & $2 \cdot 42$ & $\begin{array}{l}2 \cdot 39,2 \cdot 44 \\
P=0.001 \S\end{array}$ & $2 \cdot 41$ & $\begin{array}{c}2 \cdot 38,2 \cdot 44 \\
P=0.035 \|, \dagger\end{array}$ & $60 \cdot 66$ & $\begin{array}{l}60 \cdot 18,61 \cdot 13 \\
P<0 \cdot 001 \S\end{array}$ & $60 \cdot 49$ & $\begin{array}{l}60 \cdot 04,60 \cdot 95 \\
P=0.00211, \mp\end{array}$ \\
\hline \multicolumn{9}{|l|}{ Parental occupation } \\
\hline Manual, non-skilled ( $n$ 218) & $2 \cdot 35$ & $2 \cdot 29,2 \cdot 42$ & $2 \cdot 37$ & $2 \cdot 31,2 \cdot 43$ & $58 \cdot 69$ & $57 \cdot 58,59 \cdot 81$ & $58 \cdot 88$ & $57 \cdot 90,59 \cdot 87$ \\
\hline Manual, skilled ( $n$ 362) & $2 \cdot 31$ & $2 \cdot 27,2 \cdot 35$ & $2 \cdot 31$ & $2 \cdot 27,2 \cdot 36$ & $59 \cdot 78$ & $58 \cdot 99,60 \cdot 57$ & $60 \cdot 06$ & $59 \cdot 29,60 \cdot 82$ \\
\hline Non-manual, lower (n 200) & $2 \cdot 35$ & $2 \cdot 29,2 \cdot 41$ & $2 \cdot 36$ & $2 \cdot 30,2 \cdot 42$ & $59 \cdot 04$ & $57 \cdot 93,60 \cdot 16$ & $59 \cdot 34$ & $58 \cdot 30,60 \cdot 37$ \\
\hline Non-manual, higher ( $n$ 1218) & $2 \cdot 42$ & $2 \cdot 39,2 \cdot 44$ & $2 \cdot 41$ & $2 \cdot 33,2 \cdot 50$ & $60 \cdot 58$ & $60 \cdot 15,61 \cdot 01$ & $60 \cdot 41$ & $60 \cdot 00,60 \cdot 83$ \\
\hline Self-employed ( $n$ 93) & $2 \cdot 40$ & $\begin{array}{l}2 \cdot 30,2 \cdot 51 \\
P=0 \cdot 001 \S\end{array}$ & $2 \cdot 42$ & $\begin{array}{c}2 \cdot 39,2 \cdot 44 \\
P=0 \cdot 004 \|,+\end{array}$ & $59 \cdot 03$ & $\begin{array}{c}57 \cdot 46,60 \cdot 60 \\
P=0 \cdot 001 \S\end{array}$ & $59 \cdot 21$ & $\begin{array}{l}57 \cdot 67,60 \cdot 70 \\
P=0 \cdot 024 \|, \ddagger\end{array}$ \\
\hline
\end{tabular}

HEI, 2005 Healthy Eating Index.

$4184 \mathrm{~kJ}=1000 \mathrm{kcal}$.

†Adjusted for total HEl score, age and sex of child.

$\ddagger$ Adjusted for cost $/ 1000 €$, age and sex of child.

$\S P$ for difference between groups as calculated by ANOVA.

IIP for difference between groups as calculated by analysis of covariance.

especially when considering the existing inequalities in health among SEP groups. It also poses challenges for nutrition professionals who are providing dietary advice for diverse groups in society, since being informed about and considering the costs of different foods increases the likelihood of implementing successful dietary changes. Although the results of the study are in line with previously published research, the major disadvantage of the current study was the generalizations made when retrospectively connecting food prices to dietary data, which could have biased the results. Future studies should focus on true food expenditure, including discounts, in combination with detailed dietary intake data. It is also important to include valid SEP variables, preferably with information about disposable income.

\section{Acknowledgements}

This research was funded by the Swedish Nutrition Society. There were no conflicts of interest. P.J.R. designed the study, carried out the data collection, performed the analysis of the data and drafted the manuscript. L.H. participated in the design of the study, helped to analyse the data and helped to draft the manuscript. The authors would like to thank Agneta Hörnell and Phil Lyon for valuable comments and support.

\section{References}

1. Mackenbach JP, Stirbu I, Roskam A-JR et al. (2008) Socioeconomic inequalities in health in 22 European countries. $N$ Engl J Med 358, 2468-2481.

2. Kant AK \& Graubard BI (2007) Secular trends in the association of socio-economic position with self-reported dietary attributes and biomarkers in the US population: National Health and Nutrition Examination Survey (NHANES) 1971-1975 to NHANES 1999-2002. Public Health Nutr 10, $158-167$.

3. Lopez-Azpiazu I, Sanchez-Villegas A, Johansson L et al. (2003) Disparities in food habits in Europe: systematic review of educational and occupational differences in the intake of fat. J Hum Nutr Diet 16, 349-364.

4. Irala-Estevez JD, Groth M, Johansson L et al. (2000) A systematic review of socio-economic differences in food habits in Europe: consumption of fruit and vegetables. Eur J Clin Nutr 54, 706-714.

5. Bihan H, Castetbon K, Mejean C et al. (2010) Sociodemographic factors and attitudes toward food affordability and health are associated with fruit and vegetable consumption in a low-income French population. J Nutr $\mathbf{1 4 0}$, 823-830.

6. Mullie P, Clarys P, Hulens M et al. (2010) Dietary patterns and socioeconomic position. Eur J Clin Nutr $\mathbf{6 4}$, 231-238.

7. Dynesen AW, Haraldsdottir J, Holm L et al. (2003) Sociodemographic differences in dietary habits described by food frequency questions - results from Denmark. Eur J Clin Nutr 57, 1586-1597.

8. Boylan S, Lallukka T, Lahelma E et al. (2010) Socioeconomic circumstances and food habits in Eastern, Central and Western European populations. Public Health Nutr (Epublication ahead of print version).

9. Drewnowski A \& Eichelsdoerfer P (2010) Can lowincome Americans afford a healthy diet? Nutr Today $\mathbf{4 4}$, 246-249.

10. Carlson A, Lino M, Juan W et al. (2007) Thrifty Food Plan, 2006. Alexandria, VA: US Department of Agriculture, Center for Nutrition Policy and Promotion; available at http://www.cnpp.usda.gov/Publications/FoodPlans/MiscPubs/ TFP2006Report.pdf

11. Maillot M, Ferguson EL, Drewnowski A et al. (2008) Nutrient profiling can help identify foods of good nutritional quality for their price: a validation study with linear programming. J Nutr 138, 1107-1113.

12. Blisard N \& Stewart H (2006) How low-Income Households Allocate Their Food Budget Relative to the Cost of the Thrifty Food Plan. Washington, DC: US Department of Agriculture, 
Economic Research Service; available at http://www.ers. usda.gov/publications/err20/err20.pdf

13. Maillot M, Darmon N \& Drewnowski A (2010) Are the lowest-cost healthful food plans culturally and socially acceptable? Public Health Nutr 13, 1178-1185.

14. McDermott AJ \& Stephens MB (2010) Cost of eating: whole foods versus convenience foods in a low-income model. Fam Med 42, 280-284.

15. Kettings C, Sinclair AJ \& Voevodin M (2009) A healthy diet consistent with Australian health recommendations is too expensive for welfare-dependent families. Aust NZ J Public Health 33, 566-572.

16. Townsend MS, Aaron GJ, Monsivais P et al. (2009) Lessenergy-dense diets of low-income women in California are associated with higher energy-adjusted diet costs. Am J Clin Nutr 89, 1220-1226.

17. Cade J, Upmeier H, Calvert C et al. (1999) Costs of a healthy diet: analysis from the UK Women's Cohort Study. Public Health Nutr 2, 505-512.

18. Darmon N, Briend A \& Drewnowski A (2004) Energydense diets are associated with lower diet costs: a community study of French adults. Public Health Nutr 7 , 21-27.

19. Maillot M, Darmon N, Vieux F et al. (2007) Low energy density and high nutritional quality are each associated with higher diet costs in French adults. Am J Clin Nutr 86, 690-696.

20. Schroder H, Marrugat J \& Covas MI (2006) High monetary costs of dietary patterns associated with lower body mass index: a population-based study. Int J Obes (Lond) 30, 1574-1579.

21. Waterlander WE, de Haas WE, van Amstel I et al. (2010) Energy density, energy costs and income? How are they related? Public Health Nutr 13, 1599-1608.

22. Andrieu E, Darmon N \& Drewnowski A (2006) Low-cost diets: more energy, fewer nutrients. Eur J Clin Nutr 60, 434-436.

23. Lopez CN, Martinez-Gonzalez MA, Sanchez-Villegas A et al (2009) Costs of Mediterranean and western dietary patterns in a Spanish cohort and their relationship with prospective weight change. $J$ Epidemiol Community Health $\mathbf{6 3}$, 920-927.

24. Bernstein AM, Bloom DE, Rosner BA et al. (2010) Relation of food cost to healthfulness of diet among US women. $\mathrm{Am}$ J Clin Nutr 92, 1197-1203.

25. Burney J \& Haughton B (2002) EFNEP: a nutrition education program that demonstrates cost-benefit. $J \mathrm{Am}$ Diet Assoc 102, 39-45.

26. Goulet J, Lamarche B \& Lemieux S (2008) A nutritional intervention promoting a Mediterranean food pattern does not affect total daily dietary cost in North American women in free living conditions. J Nutr 138, 54-59.

27. Mitchell DC, Shannon BM, McKenzie J et al. (2000) Lower fat diets for children did not increase food costs. $J$ Nutr Educ 32, 100-103.

28. Raynor HA, Kilanowski CK, Esterlis I et al. (2002) A cost-analysis of adopting a healthful diet in a familybased obesity treatment program. J Am Diet Assoc 102, 645-656.

29. Stender S, Skovby F, Haraldsdottir J et al. (1993) Cholesterollowering diets may increase the food costs for Danish children. A cross-sectional study of food costs for Danish children with and without familial hypercholesterolaemia. Eur J Clin Nutr 47, 776-786.

30. Ryden P, Sydner YM \& Hagfors L (2008) Counting the cost of healthy eating: a Swedish comparison of Mediterranean-style and ordinary diets. Int J Consum Stud 32, 138-146.

31. Patterson E, Warnberg J, Poortvliet E et al. (2010) Dietary energy density as a marker of dietary quality in Swedish children and adolescents: the European Youth Heart Study. Eur J Clin Nutr 64, 356-363.

32. Drewnowski A \& Darmon N (2005) Food choices and diet costs: an economic analysis. J Nutr 135, 900-904.

33. Slimani N, Fahey M, Welch A et al. (2002) Diversity of dietary patterns observed in the European Prospective Investigation into Cancer and Nutrition (EPIC) project. Public Health Nutr 5, 1311-1328.

34. Enghardt Barbieri H, Pearson M \& Becker W (2006) Riksmaten - children 2003. Food and nutrient intake in Swedish children. Uppsala: Swedish National Food Administration; available at http://www.slv.se/upload/ dokument/rapporter/kostundersokningar/riksmaten\%20_ \%20barn_2003_livsmedels_och_naringsintag_bland_barn_i_ sverige.pdf

35. Goldberg GR, Black AE, Jebb SA et al. (1991) Critical evaluation of energy intake data using fundamental principles of energy physiology: 1. Derivation of cut-off limits to identify under-recording. Eur J Clin Nutr $\mathbf{4 5}$, 569-581.

36. Becker W, Lyhne N, Pedersen A et al. (2004) Nordic Nutrition Recommendations 2004: Integrating Nutrition and Physical Activity, 4th ed. Copenhagen: Nordic Council of Ministers.

37. Guenther PM, Reedy J \& Krebs-Smith SM (2008) Development of the Healthy Eating Index-2005. J Am Diet Assoc 108, 1896-1901.

38. Jansson A (2010) The Keyhole symbol. http://www. slv.se/en-gb/Group1/Food-and-Nutrition/Keyhole-symbol/ (accessed April 29 2010).

39. Guenther PM, Reedy J, Krebs-Smith SM et al. (2007) Development and Evaluation of the Healthy Eating Index2005: Technical Report. Alexandria, VA: US Department of Agriculture, Center for Nutrition Policy and Promotion; available at http://www.cnpp.usda.gov/Publications/HEI/ HEI-2005/HEI-2005TechnicalReport.pdf

40. Swedish National Food Administration (2010) The food database. http://www7.slv.se/livsmedelssok/sok.aspx?lang $=2$ (accessed April 2010).

41. Kennedy E (2008) Putting the pyramid into action: the Healthy Eating Index and Food Quality Score. Asia Pac J Clin Nutr 17, Suppl. 1, 70-74.

42. Nordenskiöld T (2010) Rekordår för ICAhandlarna (Banner year for ICA). Dagens Industri, 16 February.

43. Market (2010) Ojämn kamp om barnfamiljerna (The battle for families with children is uneven). http://www.market.se/ Brancher/Dagligvaror/Ojamn-kamp-om-barnfamiljerna/ (accessed May 2010).

44. Murakami K, Sasaki S, Okubo H et al. (2007) Monetary costs of dietary energy reported by young Japanese women: association with food and nutrient intake and body mass index. Public Health Nutr 10, 1430-1439.

45. Rolls BJ, Drewnowski A \& Ledikwe JH (2005) Changing the energy density of the diet as a strategy for weight management. J Am Diet Assoc 105, 5 Suppl. 1, S98-S103.

46. World Cancer Research Fund/American Institute for Cancer Research (2007) Food, Nutrition, Physical Activity, and the Prevention of Cancer: A Global Perspective. Washington, DC: WCRF/AICR; available at http://www.dietandcancerreport. org/

47. Duffey KJ, Gordon-Larsen P, Steffen LM et al. (2009) Regular consumption from fast food establishments relative to other restaurants is differentially associated with metabolic outcomes in young adults. J Nutr 139, 2113-2118.

48. Drescher LS, Thiele S \& Mensink GBM (2007) A new index to measure healthy food diversity better reflects a healthy diet than traditional measures. J Nutr 137, 647-651.

49. Wadman C, Boström G \& Karlsson A-S (2008) Health on Equal Terms? Results from the 2006 Swedish National 
Public Health Survey. Östersund: Swedish National Institute of Public Health; available at http://www.fhi.se/ Documents/Statistik-uppfoljning/Folkhalsoenkaten/Resultatarkiv/Rapporter/A2008-1-health-on-equa-terms.pdf

50. Inglis V, Ball K \& Crawford D (2009) Does modifying the household food budget predict changes in the healthfulness of purchasing choices among low- and high-income women? Appetite 52, 273-279.

51. Kirkpatrick S \& Tarasuk V (2003) The relationship between low income and household food expenditure patterns in Canada. Public Health Nutr 6, 589-597.

52. Giskes K, Van Lenthe FJ, Brug J et al. (2007) Socioeconomic inequalities in food purchasing: the contribution of respondent-perceived and actual (objectively measured) price and availability of foods. Prev Med 45, 41-48.
53. Turrell G, Bentley R, Thomas LR et al. (2009) A multilevel study of area socio-economic status and food purchasing behaviour. Public Health Nutr 12, 2074-2083.

54. Livingstone MB \& Black AE (2003) Markers of the validity of reported energy intake. J Nutr 133, Suppl. 3, 895S-920S.

55. Lallukka T, Pitkaniemi J, Rahkonen O et al. (2010) The association of income with fresh fruit and vegetable consumption at different levels of education. Eur J Clin Nutr 64, 324-327.

56. Monsivais P \& Drewnowski A (2007) The rising cost of lowenergy-density foods. J Am Diet Assoc 107, 2071-2076.

57. Glanz K, Basil M, Maibach E et al. (1998) Why Americans eat what they do: taste, nutrition, cost, convenience, and weight control concerns as influences on food consumption. J Am Diet Assoc 98, 1118-1126. 